\title{
Uterine Leiomyosarcoma: a Management Dilemma
}

\author{
Sunita Gupta, Geetika, Rani Bansal', Himanshu Chauhan² \\ From the Department of Obstetrics and Gynecology; Department \\ of Pathology'; Department of Anaesthesia and Critical care ${ }^{2}$; \\ Subharti Institute of Medical Sciences, Meerut, India.
}

\begin{abstract}
:
Uterine leiomyosarcoma (ULMS) are rare and aggressive smooth muscle tumours accounting for approximately $1 \%$ of patients with uterine cancer. ULMS are neoplasm's of high metastatic potential. Diagnosis of leiomyosarcoma is often made after hysterectomy as symptoms and signs are nonspecific and imaging methods fail to differentiate it from benign conditions. Trans (abdominal) hysterectomy was performed in a 38 year old female with a provisional diagnosis of fibroid uterus. Histopathology showed leiomyosarcoma stage 3A. Patient refused for chemotherapy and developed recurrence in 6 months and succumbed after 3 months of recurrence. ULMS continue to provide a major challenge for treatment of the primary presentation and for relapses.
\end{abstract}

Key words: Uterine Neoplasms, Leiomyosarcoma, Adjuvant Chemotherapy, Smooth Muscle Tumor, Leiomyoma.

\section{Introduction}

Uterine leiomyosarcoma (ULMS) are rare smooth muscle tumours accounting for approximately one percent of patients of all uterine malignancies and represent the second more common uterine sarcoma [1,2]. Generally uterine fibroids do not develop into malignant leiomyomas [3] but leiomyosarcomas frequently coexist within a fibroid uterus. Diagnosis of leiomyosarcoma is often made after hysterectomy, as clinical diagnosis is usually of benign fibroid. Approximately $0.5 \%$ of women who have hysterectomies for uterine fibroid are found to have leiomyosarcomas [4]. Median age of presentation of ULMS is usually between 47 to 56 years [5,6]. We present a case report of this rare tumour arising from uterus.

\section{Case Report}

A 38 years female, Para-4, Abortion-0, was admitted with complains of continuous bleeding per vagina, pain and swelling in abdomen for three months. She had menorrhagia for two years. On clinical examination uterus was enlarged, equivalent to 20 weeks gestation, firm and tender with restricted mobility. Bilateral adnexa were normal. Her haemoglobin was $4.7 \mathrm{gm} \%$ \& blood group was B positive. Ultrasonography showed a large

Corresponding Author: Dr. Sunita Gupta

Email: drsunitachauhan@gmail.com

Received: January 18, 2013 | Accepted: March 20, 2013 | Published Online: May 10, 2013

This is an Open Access article distributed under the terms of the Creative Commons Attribution License (creativecommons.org/licenses/by/3.0)

Conflict of interest: None declared | Source of funding: Nil | DOl: http://dx.doi.org/10.17659/01.2013.0033 
heterogeneous hypo echoic mass lesion of size $87 \times 74 \mathrm{~mm}$ in the posterior wall of the uterus. Endometrium was normal with no adnexal mass. X-ray chest was normal. Cervical cytology and endometrial biopsy were negative for any malignant pathology. A provisional diagnosis of fibroid uterus with degeneration was made and her total (abdominal) hysterectomy with bilateral salpingo-oopherectomy was done after transfusing six units of blood. Per-operatively uterus was found to be irregularly enlarged and few omental adhesions to uterus were present. Pelvic and para aortic lymph nodes were palpated and no enlarged lymph node was found. A small nodule present in uterovesical fold was resected. Specimens were sent for histopathological examination. Her postoperative period was uneventful.

Histopathological examination of the specimen showed leiomyosarcoma involving whole of the myometrium \& endometrium with mitotic index >9-10/ high power field and coagulative tumour cell necrosis with cellular atypia [Fig.1]. Cervix and both adnexal structures had normal histology while resected nodule showed tumour cells. Patient and her attendant were counselled regarding the risks and benefits of radiotherapy and chemotherapy. Because of economic constraint they refused for further management. After 6 months patient develops pelvic recurrence and succumbed within 3 months of recurrence.

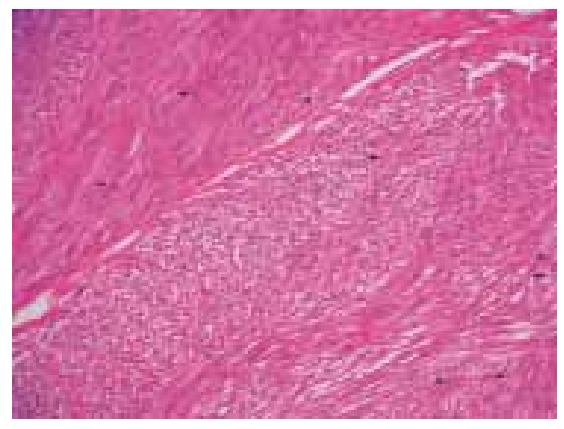

Fig.1: Histopathological microscopic examination showing a cellular tumor arranged in interlacing bundles of spindle cells with elongated hyperchromatic nuclei and nuclear pleomorphism

\section{Discussion}

Preoperative diagnosis of leiomyosarcoma is a challenge for the gynaecologist as signs and symptoms are nonspecific and imaging methods are not able to differentiate it from other benign conditions. Histological diagnosis by endometrial sampling gives a low sensitivity of approximately $30 \%$ [4]. Surgical treatment of the patient with ULMS of the uterus includes extra facial total (abdominal) hysterectomy with or without salpingo-opherectomy. Prognostic indicators of the disease are age, stage of disease, tumour size, mitotic index, tumour cell necrosis and depth of tumour invasion in myometrium. The recurrence rate is approximately $70 \%$ for stage I and II disease, and the site of recurrence is often distant. Postoperative treatment of uterine sarcomas has been long debated. Role of lymphadenectomy appears to be limited, because the incidence of lymph node metastasis is very low in the absence of extrauterine disease. A routine lymph node dissection is not required in women with disease confined to uterus with normal lymph nodes on observation and palpation, as patients with negative lymph nodes may develop recurrence at distant sites [6]. Adjuvant pelvic radiotherapy appears to improve local control without any significant impact on overall survival. There is little evidence 
in the literature supporting the use of adjuvant chemotherapy in any gynaecological sarcomas except for carcinosarcoma. Uterine sarcomas have a high tendency to develop distant recurrences, and recent data on adjuvant chemotherapy in soft tissue sarcomas are promising. In a Swiss study, the combination of ifosfamide and doxorubicin obtained similar response rates in advanced gynaecological sarcomas and in advanced soft tissue sarcomas of other sites [7]. However, in a small phase II study, four cycles of adjuvant chemotherapy with gemcitabine and docetaxel in patients with completely resected stage I-IV, high-grade uterine leiomyosarcomas resulted in 2-years of progression free period in $45 \%$ of the patient with a median of progression free survival of 13 months [8]. For recurrent disease no curative option is currently available with the possible exception of surgery for lung and hepatic metastases. Gemcitabine has been shown to have a role in persistent or recurrent ULMS and when combined with docetaxel, the overall response rate has been shown to be 53\% [8]. Early tumor stage, age at diagnosis $<50$ years, absence of vascular space invasion, low myometrial invasion and low histologic grade are parameters of a good prognosis in women with uterine leiomyosarcoma [9]. Early diagnosis and complete surgical removal of the tumour is still the only treatment to improve overall survival of the patient.

\section{Conclusion}

Mostly leiomyosarcomas are diagnosed at advanced stage and has poor response to chemotherapy and radiotherapy. Further development of imaging techniques, biochemical and molecular marker is needed to diagnose it at early stage.

\section{References}

1. Ramondetta L, Bodurka D, Deavers M, Jhingran A. Uterine Sarcomas. In: Eifel PJ, Gershenson DM, Kavanagh JJ, Silva EG (eds). M. D. Anderson Cancer Care Series, Gynecologic Cancer. Springer: New York; 2006. pp.125147.

2. Zaloudek C, Hendrickson MR. Mesenchymal tumors of the uterus. In: Kurman RJ (eds). Blaustein's pathology of the female genital tract. New York: Springer-Verlag, Inc; 2002. pp.561-615.

3. Quade BJ, Wang TY, Sornberger K, Dal Cin P, Mutter GL, Morton CC. Molecular pathogenesis of uterine smooth muscle tumors from transcriptional profiling. Genes Chromosomes Cancer. 2004;40:97-108. doi:10.1002/ gcc. 20018

4. Leibsohn S, d'Ablaing G, Mishell DR Jr, Schlaerth JB. Leiomyosarcoma in a series of hysterectomy performed for presumed uterine leiomyomas. Am J Obstet Gynecol. 1990;162:968-974.

5. Giuntoli RL 2nd, Metzinger DS, DiMarco CS, Cha SS, Sloan JA, Keeney GL, et al. Retrospective review of 208 patients of leiomyosarcoma of the uterus: Prognostic indicator, surgical management and adjuvant therapy. Gynecol Oncol. 2003;89:460-469. doi 10 .1016/ S0090-8258(03)00137-9,

6. Wu TI, Chang TC, Hsueh S, Hsu KH, Chou HH, Huang HJ, et al. Prognostic factors and impact of adjuvant chemotherapy for uterine leiomyosarcoma. Gynecol Oncol. 2006; 100:166-172. Doi 10.1016/i.ygyno 2005.08.010

7. Gadducci A, Cosio S, Romanini A, Genazzani AR. The management of patients with uterine sarcoma: a debated clinical challenge. Crit Rev Oncol Hematol. 2008; 65:1 29-142.

8. Hensley ML, Ishill N, Soslow R, Larkin J, Abu-Rustum N, Sabbatini P, et al. Adjuvant gemcitabine plus docetaxel for completely resected stages I-IV high grade uterine leiomyosarcoma. Results of a prospective study. Gynecol Oncol. 2009; 1 1 2:563-567.

9. Bodner K, Bodner-Adler B, Kimberger O, Czerwenka K, Leodolter S, Mayerhofer K. Evaluating prognostic parameters in women with uterine leiomyosarcoma. A clinicopathologic study. J Reprod Med. 2003;48:95-100. 